\title{
Best Practices for Motivation and their Implementation in the Greek Public Sector for Increasing Efficiency
}

\author{
Dr Rossidis loannis (PhD MSc, MBA,BA) \\ Functionary in the Ministry of Finance, Lecturer at the University of the Aegean \\ Email: jrossidis@aegean.gr
}

\section{Dr Aspridis George (PhD)}

Associate Professor in Business Administration, University of Applied Sciences of Thessaly/TEl of Thessaly Email: aspridis@teilar.gr

\section{Dr Blanas Nikolaos (PhD)}

Associate Professor in Business Administration, University of Applied Sciences of Thessaly/TEl of Thessaly Email: nikoblanas@teilar.gr.

\section{Bouas Konstantinos (MPA, NCPA, BA)}

Policy Analyst, Institute for Regulatory Policy Research, Ministry of Administrative Reform \& E-government Email: bouaskostas@gmail.com

Katsimardos Petros, (MPA, NCPA, BA)

Policy analyst, Institute for Regulatory Policy Research Ministry of Administrative Reform \& E-government Email: katsimardospetros@yahoo.gr

Doi:10.5901/ajis.2016.v5n3s1p144

\section{Abstract}

The contemporary socio-economic conditions and the constantly increasing requirements for higher growth rates, shape the eternal need for improvement of the administrative function of the public sector. The current problem of the Greek public administration, questions the productivity of human resources. This problem tends to distend excessively in today's "memorandum era", because of the unexpected pay cuts, the stagnation of the grade promotions and the all in all work disappointment caused by the economic crises. An extremely useful management answer to this problem is given by the science of organizational behavior, through the theory of the instigation of the personnel. However, in the case of the Greek public administration, this theory loses part of its capacity, since it cannot provide financial incentives (because of its existing budgetary restrictions). Consequently, this analysis is focused on the improvement of productivity in human recourses based on non-financial incentives, which do not affect directly the public economy. The main mission of this study is to investigate the instigation methods adapted by the Greek public administration, for the improvement of the public sector's performance. The methodological practice that will be used for the documentation of the above case, is the comparative benchmarking between public and private sector, following the already used instigation model suggested by the business management. During the study, the optimal motivation practices used by the private sector will be found, analyzed and then applied to the public sector. The main aim of this study is to increase the efficiency of the public sector, supplying it with useful modern business tools for the general functional improvement of the public administration.

Keywords: Motivation, Public Administration, Public Servants, Efficiency

\section{Introduction}

In the recent years, the constantly increasing problems the public-sector faces, demonstrate the need for a change. Immoderate bureaucracy, corruptness, mismanagement, low efficiency and introversion are only some of the problems the public sector must solve. The predominant operational rationality insists that Government and the well-developed 
private sector, must join hands. The functional improvement of the public sector constituted one of the most central management matters of the $20^{\text {th }}$ century, since it is directly connected with the socio-economic growth.

Greece presents an exceptionally unlimber and over-loaded public sector, with dominant characteristics its inefficiency, high levels of bureaucracy and misconduct. The State administration must improve the public sector using essential programs of management development, so that it will be capable of following both the international developments and its national mission. One of the current problems the Greek public sector faces is about the productivity of human resources. This problem tends to distend excessively in today's "memorandum era", because of the unexpected pay cuts, the stagnation of the grade promotions and the all in all work disappointment.

This study will attempt to develop individual tools from the organizational behavior science, which are used by the private sector and they can be very useful to the public sector as well. This approach will focus on the precious tool of instigation, without paying attention to any financial incentives (reflecting on the budgetary restrictions Greece faces).

\section{The Theory of Instigation Serving as A Wheel for the Increase of Productivity}

A particularly critical factor for the right management of the personnel, constitutes the power of management to convince its employees to practice their duties in the desirable efficiency level (Denrardtetal, 2009). This practice can be achieved, when management can determine the suitable system of incentives (Roseetal, 1999). The significance of instigation according to V. Berlson and G. Steiner, is reported in "an internal situation that activates and directs the human behavior towards the main objectives". The instigation aims to activate a desirable activity with the exchange of satisfaction gained from the fulfilling of one, or more than one, necessities. In professional level, the need satisfaction can be reported in the increase of economic rewards, other benefits, improvement of grading position, hierarchical evolution, bigger prospects, reduction of pressure or worktime, improvement of the social status, enrichment of individual skills etc.

The incentives used for the motivation of employees, were included in an abundance of theories. The most important models developed, are distinguished in the following two categories. The first category includes the theories looking for the content of instigation. Some of the most known theories include A. Maslow's studies about the hierarchy of necessities, E. Mayo's about human relationships, F. Hersberg's and others. The second category includes the theories which show the process of instigation. These theories develop the instigation system of the personnel and the emerged relationships. The most representative theories are those of Porter, Lawler, Vroom and others (Gomez-Mejia et al, 2015; Noe et al, 2009).

Now, it is crucial to mention certain instigation techniques used by the operational management (Kreitner, 2009), which set an example for the public sector; These include :

a. Relation between efficiency and remuneration. Under this practice both high efficiency and work effort of the employee, are recognized and rewarded with various benefits, such as :

- Financial incentives. The remuneration increase is the most popular and efficient type of incentives. The price incentive serves as a recognition of the employee's efforts and is related to the incentives of safety, success, prestige, social recognition etc. Financial incentives are provided in relation to productivity, the achievement of predetermined goals, the qualitative transaction of duties, the permanent attendance at the working place, the participation in the process of improvement and the general behavior.

- Common Incentives The common incentives aim to support the collective effort of employees, preventing any competitive action caused by individual incentives. At this type of incentives, the main attempt is the connection between productivity and total result of the organism.

- Participation Incentives in Corporate Governance. In this case, management places the employee in the position of the director, identifying the employee's interest with that of the organization. One popular strategy to follow is the distribution of shares to the personnel, so that the productive disposal of the employees will follow the company's course.

b. Participation in Decision-making. A modern management system of incentives includes the opinions of the employees in the final decision-making process. This system aims to increase personnel's sense of responsibility and improve its total efficiency (Bourantas, 2002). The system's individual practices of achievement are supported by autonomous/semi-autonomous work teams and by quality circles.

- At the range of the autonomous/semi - autonomous working teams, the work analysis is based on the team and not on the individual. Namely, every work task is considered as a unique project that is assigned to all the employees, causing an endogenous interaction. At the same time, responsibility and power are allocated to all the members of the team, so that a spirit of collegiality is cultivated. 
- The Quality Circles, are circles of voluntary participation from part of the organization's personnel, aiming to detect problems or activating opportunities for partnership affairs, via conferences. Some of the individual goals set by the quality circles, include the quality upgrade, the increase of productivity, the improvement of the service level, the improvement of work conditions, the communication development, the improvement of organizational structures, the reformation of control systems etc. Through this system, the employees acquire a powerful incentive for participation in the corporate joints, while at the same time, a complementary management support is provided in the management sector, increasing the administration's efficiency.

c. Designing appropriate work positions. It is a fact that one of the most important criteria for a work choice, concentrates at the work position's profile (content and basic characteristic). Consequently, the profits of a well-designed work position, constitute powerful incentives for work efficiency. According to the model of Hackman and Oldham, the crucial elements that a work position must contain, so that a beneficial instigation of the personnel will be achieved, include variety and importance of its duties (pointing here the sense of selfesteem and creativity), development prospects, autonomy (strengthening the sense of responsibility) and feedback. These characteristics, can increase the personnel's efficiency, providing a powerful incentive for productivity and professional consequence.

d. Leaders Behavior. The motivation capability of the prevailing leaders, plays an exceptionally important role. As a "leader", is comprehended the hierarchical superior, who inter alia owes to manage his subordinates, so that both productivity and personnel's satisfaction are achieved. The leader serves a double role, first as the final representative of the system of incentives set by the business administration, and second as the natural leader who must inspire his/her personnel so it will function at the desirable efficiency. The leadership of the superior and his capability to fairly divide the total amount of work into individual responsibilities, will shape the disposal of the personnel so that it will implement efficiently its duties. At the same time, leader's behavior towards the team members, who shape the final outcome, is of a great importance. Consequently, there must be a very careful look at the leader's attitude, since his/her duty is to inspire the employees to make a greater effort for the best outcome.

e. The Benchmarking of Outcomes as an Instigation Practice. This system of incentives applies to standardized results per group of people or individual, evaluating the employees' effort, using as benchmark the predetermined model. In this way, the group of people/individual get to know the main goal and the criterion of evaluation, while the business allocates an explicit picture about the performance of the personnel. In the administration and the personnel's interest, the participation of the employees is pursued during the process of defining the incentives. Consequently, the goals should be possible to achieve and the personnel must not feel any pressure. The administration is obliged to determine the personnel's actual production capacity, pointing to the exclusion of delusions caused by unfeasible outcomes. Based on this motivation system, which is included in the theories of "Management By Objectives" and "Efficiency Management", the instigation of the personnel is attempted and there is also an engagement for execution of the anticipated objectives. Through this process, the tasks and responsibilities of the personnel are classified, there is an increase in human resource's productivity, a comprehension of the business structures, a highly effective co-ordination and support in the remaining management operations (Aspridis, 2013).

It should be mentioned that for the efficient instigation of the personnel, a great number of sciences such as psychology, sociology and anthropology along with the organizational behavior theory (which constitutes a combination from a variety of sciences), and the individual techniques that affect the employees' senses, come together in order to improve the efficiency level of the personnel (Aspridis, 2012). Following the example of the private companies, which follow the above techniques, public bodies could upgrade the way they function, improving their level of efficiency.

\section{The Development of the Public Servants' Instigation Tools Via the Comparative Analysis, Using Methods of The Private Sector}

The comparative analysis of the instigation methods among public and private sector, both shows obvious differences and individual convergence elements, which, through the right choice of policies, can support the public sector, approaching the efficiency levels of the private sector. In this analysis, important differences that discriminate public from private sector, (such as legislative restrictions, public sector's privileges, private sector's profit parameter, etc.), must be included. 
The following results of individual researches, which focus on the comparative analysis of instigation methods between public and private sector, aim to highlight the main differences found in the focus points of instigation policies. Based on the survey conclusions of Frank Sue and Lewis Gregory, public servants pay more attention on the work safety in the public sector, compared to the private employees (Frank et al, 2004). The main interest of public servants, contrary to that of the private employees, serves the society and the public interest without ensuring any promotions.

According to Buelens and Van der Broeck's research, private employees give far more value on the economic rewards (Buelens et al, 2007). The economic rewards constitute a powerful productivity incentive for private employees, mostly for senior management, while public servants do not relate productivity with financial incentives. Based on this research, public servants are influenced to a great degree by internal incentives, like the sense of duty and self-fulfillment. Public servants show more preference in safe and consistent working environments, with a warm and harmonious atmosphere based on reciprocity and respect. The research constitutes that the differences in individuals' instigation method are mostly based on their working field and less on demographic elements. The internal incentives, mostly the ones that have nothing to do with economic rewards, are more valuable to the public servants.

Despite the low productivity level of the Greek public sector, public administration does not follow any clear instigation policy. The working elements about the professional life of public servants, does not show any essential productivity incentive. It is a fact that work efficiency is not connected to any grading, wage or other incentive. Consequently, there is no reason to increase work intensity, so that the qualitative work process is based on the personnel's self-conscientiousness. According to Bourantas et al (1999), in which the defining factors of work efficiency are described, the human resources gap between public and private sector, is clearly presented. This study used a sample of 1.150 employees working at different Ministries, Public Organisms, Public and Private Enterprises (Bourantas et al, 1999). According to the article, some factors affecting the existing instigation gap, except that efficiency and economic rewards are not related, are included:

- In the characteristics of the work content, mainly in the variety and importance of duties, in the autonomy of implementation, in information about the outcomes, in the confusion and conflict about roles, policies and processes.

- In the general lack between the efforts - efficiency - remuneration connection.

- In the general atmosphere of the public administration, with unrecognized efforts, no stability in efficiency levels, and political intervention.

- In the quality of leadership by directors of all hierarchical levels and mainly in the operational levels of remunerations and orientation of energies in order to achieve the objectives.

At this point, before any technical upgrade on the methods of instigation of the personnel, a very crucial problem should be solved. This problem includes the assessment of public service's efficiency, and it mostly focuses on the individual departments and personnel. The assessment's issue is quite difficult and at the same time of great importance, since it constitutes part of public servants' productivity level and relates to the effectiveness of the public sector. The absence of reliable assessment of public servants, impedes the identification of weaknesses and the rational management of the human resources. During the past, ineffectual and unworthy administrative legislations lead to quite many reformation efforts about the assessment of public servants (Academy Athens, 1998). Consequently, the main concern of public administration, must be the recording analysis of the personnel's existing productivity. In addition, the regions that need improvement are detected, and the formation of a management strategy of human recourses in formed, targeting on the total upgrade of the personnel. All the tools of human resources management, including the system of intakes, remunerations, developments, evaluations, transfers and the system of education, should be modified in order to respond on the needs of flexibility and adaptability without ignoring the existing budgetary restrictions (Katsimardos et al, 2012). The assessment will present the real picture of the personnel and reveal the problematic focus points.

Following the solution of the above issue, the administration will be capable of using specialized business management tools, in order to improve efficiency levels. The instigation technique, mostly used in the private sector (high levels of success), offers to the public administration, a powerful system of incentives, supporting the increase of productivity. Based on the above analysis, the public servant does not show any need, demand, or work enhancement and therefore he is presented as incurious towards the implementation of his/her duties. The instigation of the personnel constitutes a very important management problem, related to the productivity of human recourses. Connecting efficiency incentives with financial and grading ones, involve great improvement in the productivity of employees. The two basic disincentives related to the work concentration of the employees constitute the static salaries and the employees' hierarchical evolvement. The previous two disincentives, could develop in a positive way the already existing system, offering to the employees an alternative chance to increase their remuneration and upgrade their professional career. 
Nowadays, the exploitation of financial incentives is a very difficult process, since there are budgetary restrictions that prevent the use of economic compensations for the improvement of productivity. Consequently, it is obligatory to exploit different types of incentives, as follows (Gomez-Mejia et al, 2015; Noe et al, 2009):

- The common incentives, which aim to strengthen the teamwork spirit missing from the public sector.

- The participation incentives in decision-making, which encourage the reduce of work alienation experienced by the public employees, as being part of a giant problematic State apparatus productivity process. At the same time, State Administration could attain a supporting management tool used from the nearest to the existing problem appropriate department.

- The design incentives for adapted work positions, which promote the unique skills of the personnel, create prospects of development and increase the employees' satisfaction offering them suitable work positions. It's a fact that due to the existing recruitment system, public servants rarely occupy work position which correspond to their skills and potentials.

- The incentives formed by the results of benchmarking. This type of incentives reflects the lack of an essential evaluation system in the public sector. The benchmarking of the results, define the standards of improvement. One typical example is the case of the new public servants, who initially work very professionally, but since their efforts are not recognized and they do not receive any other benefits, soon give up.

A research carried out by OECD, presented the tendencies that concern labor costs in public sector based on efficiency (Performance-Related Pay), and examined a) the financial reward offered to every individual or group of employees based on their productive potential and b) the resulting productivity development (in the website: www.oecd.org). This research, supports the policy where remuneration is performance related, a policy recently growing in the public administration sector of the countries of OECD. At the recent past, the countries of OECD increased their employees' salary based on how many years they worked for the company and not proportionally to their efficiency level. The policy change came due to the need for reformation of the existing instigation techniques, the focus on the administrative financial reward and the recognition of the work effort and devotion. The new system of payment based on the efficiency level, leads to the increase of competitiveness, using as role model the private sector. The first countries that adapted this rewarding system, were the governments of Canada, Denmark. Holland, New Zealand, Sweden, United Kingdom and USA, followed by an abundance of other countries. About two thirds of OECD countries have in some degree adapted for their public services, payment based on the efficiency system. The countries of Denmark, Finland, Korea, New Zealand, Switzerland and United Kingdom have made this policy system official. The countries of Canada, Ireland, Italy and Norway have applied this system in a managerial level, while the countries of Ireland, Norway and France are still in an experimental level (at a ministry level). Some of the current tendencies include:

- The standardized systems where payment is related to efficiency, which are developed into decentralized systems in countries like Denmark, New Zealand, United Kingdom and USA.

- The expansion of this rewarding system, from the superior administrative executives - to the personnel.

- The current trend encouraging many countries to focus on togetherness and collegiality.

This particular rewarding policy can operate as a very useful instigation tool or even as a wheel for a) the establishment of more flexible work methods, b) the promotion of efficient methods of complete tasks and staff management and c) after the incorporation of new technologies resulting in renovated means of work. Some new elements define future management trends, as follows:

- Significance should be given in the validity of the assessment process of performance, since it constitutes the main core of the rewarding system.

- Attention should be given in the exploitation of this system, so that it will function additionally to the remaining management methods of the personnel.

- Both the culture of each organization and the culture of each country should be valued before the planning of the rewarding policy, where payment is related to efficiency.

- There should be prevention of any resulting problem through the coordination of the personnel, the forecasting of expenses etc.

- Remunerations based on efficiency, should correspond to how the human resources management is distributed for regulation.

At this point it should be stressed that a system of financial incentives which is based on the employees' efficiency level, it does not necessarily mean that it will increase the labor cost. An income redistribution policy that will reward the more productive employees, is more likely to happen, supporting the meritocratic staff evaluation, focusing on the quality and the efficiency of the provided services. The performance improvement of the public sector will be benefited as well. 
A very popular instigation method, helps the personnel of a public organization to embrace the main operational vision, changing a common objective to an individual one. In order to encourage this policy, instigation practices and advanced communication methods are combined so that the employees will be convinced about the importance to participate in the achievement of a high public interest and a common objective both very useful for the entire group and each individual.

Another tool of the operational management, very helpful to the instigation process, is the "leadership" practice. Public administration is a multi-level hierarchical system, the executive members of which, progressing based on their age. The structure of this bureaucratic system promotes a big number of bureaucratic executives - who are mostly interested in completing their personal tasks rather than completing the common duties of the department. The executives do not consider as their duty the development of their subordinates, showing no interest either for the education and training of their personnel nor for their encouragement and their psychological support, resulting to a management discontinuity in the organism. Though, it's a fact that in public sector the superior administrative executives are unable to function either as managers or as leaders. Asking to such an executive, who has not even realized his/her administrative tasks, to act as a leader, would be at least an exaggeration. The increased added value the public administration would have, in case its executives were also leaders, is very important to be mentioned, since it would create prospects of a) implementation of public policies in the most effective way, b) exploitation of the employees in the most productive way c) enrichment of the operations of the organism with the use of new, innovative practices etc. The creation of future leaders is an important process both for public and private sector, but the public sector has not realized it yet. The development of leaders along with an applied pithy policy of incentives, can both be very helpful for the productivity of human resources and the improvement of the total efficiency level of public administration.

\section{Conclusions}

Greece, which is bound by memorandum obligations, is called to improve the levels of productivity of its civil servants, under the budgetary restrictions caused by economic crisis. The motivation of human resources in the public sector, constitutes a very difficult and at the same time exceptionally important task, which can lead to particularly high levels of efficiency. The motivation of employees in the public sector, along with many other parameters, such as the socio economic conditions, the culture and customs in each country, every country's constitutional system or even the broader sense of an entire State (Vandenabeele, 2007), constitutes a multidimensional process (taking into account the peculiar nature of the State). The private sector example is used as a specific "guide", including the most optimal practices suggested to the public sector, covering the emerging void of administrative expertise that derives from public bureaucracy. The extremely insufficient evaluation system, the budgetary restrictions and the absence of working interest, perhaps constitute the most basic problems of the personnel's productivity in the Greek public administration. However, the very peculiar nature of these specific problems can direct the state administration into the right direction, presenting the suitable motivation policies which can lead to the improvement of the employees' productivity.

The public administration could strengthen its operational force, applying different motivation methods, as follows :

- One modern, meritocratic and efficient evaluation system that will benefit the best businessmen, offering them hierarchy and salary incentives (Performance Related Pay - without any increase, but rather a reallocation of the operating costs), which can greatly motivate the Greek personnel.

- Redesigning the work positions, can help as a valuable evaluation of the active and inactive employees, by modernizing the existing organizational structure so that the human resources will be better managed.

- The participation of employees in the decision-making process, serving as an opportunity for those regulating the administrative problems (first line), suggesting solutions to support the given inefficient central administration.

- Using common incentives for team work focusing on the arise of future leaders.

- Applying channels of communication for the promotion of a new culture in the work of civil servants, based on which the promotion of the public interest will be very important, developing a common conscience. Another indicative factor is the State's functional improvement, which involves the improvement of the personal position for both the employees and all the Greek citizens.

Even though, Greece is on an extremely difficult position, it pledges to succeed extraordinary administrative changes, by improving its personnel's productivity level (reformation of the public sector). The highly developed private sector and a better functioning of the public administration, can be examples for the public sector helping it to cover the lack administrative expertise, strengthening the Greek economy. 


\section{References}

Academy of Athens (1998). The Public Administration in front of the 21st century challenge. Athens [in Greek].

Aspridis, G., (2013). Introduction to Civil and Administrative Organization of the Greek State, Athens : Propobos [in Greek].

Aspridis, G., (2012). Alternative ways to motivate employees in crisis. The effect of music on enhancing worker effectiveness. Proceedings of MIBES, 25/27-12-2012 (23 - 38), Larissa [in Greek].

Bourantas, D. (2002). Management, Athens : Benos [in Greek].

Bourantas, D., and Papalexandris, N. (1999). Personality traits discriminating between employees in public and in private-sector organizations. The International Journal of Human Resource Management. Vol. 10, Issue 5.

Buelens M., and Van den Broeck H. (2007). An Analysis of Differences in Work Motivation between Public and Private Sector Organizations. Public Administration Review. Vol. 67, No. 1.

Dessler, G. 2012. Human resources management - Basic concepts and modern trends. Athens : Kritiki [in Greek].

Denrardt, R.,and Denhardt, J. (2009). Public Administration. An Action Orientation. Belmont: Thomson Wadsworth.

Frank, A., and Lewis B. (2004). Government Employees: Working Hard or Hardly Working? The American Review of Public Administration. Vol. 34, No. 1.

Gomez-Mejia, R. L., Balkin, B. D., and Cardy, L. R., (2015). Managing Human Resources, Nikosia : Broken Hill Publishers LTD [in Greek].

Katsimardos, P., and Bouas, K., (2012). A new strategy for the management of human resource in public administration, Athens : Regulatory Policy Research Institute ([online] http://www.inerp.gr/el/blog/60-nea-stratigiki-anthropinon-poron-dimosiasdioikisis.html, accessed on 8 December 2016) [in Greek].

Kreitner, R. (2009). Management. Boston : Houghton Mifflin Harcourt.

Kressler, H. (2003). Motivate and Reward. Performance Appraisal and Incentive Systems for Business Success. New York : Palgrave Macmillan.

Noe, R., Hollenbeck, J., Gerhart, B., and Wright P. 2009. Human Resources Management: Gaining a competitive advantage. Athens : Papazisis [in Greek].

Rose, A., and Lawton, A. (1999). Public Services Management. Essex: Pearson Education Limited.

Rossidis, l., (2014). Applications of business management in the Greek civil service. Athens : Stamoulis [in Greek].

Starling, G. (2010). Managing the Public Sector. Boston: Wadsworth

Vandenabeele, W. (2007). Toward a public administration theory of public service motivation: An institutional approach. Public Management Review. Vol. 9, No. 4.

www.oecd.org, [accessed on 8 December 2016]. 